\title{
COMPLEXITY OF COUNTABLE CATEGORICITY IN FINITE LANGUAGES
}

\author{
ALEKSANDER IVANOV
}

\begin{abstract}
We study complexity of the index set of countably categorical theories and Ehrenfeucht theories in finite languages.

S.Lempp and T.Slaman proved in [7] that indexes of decidable $\omega$-categorical theories form a $\Pi_{3}^{0}$-subset of the set of indexes of all computably enumerable theories. Moreover there is an infinite language so that the property of $\omega$-categoricity distinguishes a $\Pi_{3}^{0}$-complete subset of the set of indexes of computably enumerable theories of this language. Steffen Lempp asked the author if this could be done in a finite language. In this paper we give a positive answer (see Section 4). The crucial element of our proof is a theorem of Hrushovski on coding of $\omega$-categorical theories in finite languages (see [3], Section 7.4, pp. 353 - 355). Since we apply the method which was used in the the proof of this theorem, we present all the details in Section 1. Sections 2 - 3 contain several other applications of this theorem. In particular in the very short Section 2 we give an example of a non-G-compact $\omega$-categorical theory in a finite language. In Section 3 we show that there is a finite language such that the indexes of Ehrenfeucht theories with exactly three countable models form a $\Pi_{1}^{1}$-hard set. Here we also use the idea of Section 4 of [7] where a similar statement is proved in the case of infinite languages.

The main results of the paper are available both for computability theorists and model theorists. The only place where a slightly advanced model-theoretical material appears is Section 2. On the other hand the argument applied in this section is very easy and all necessary preliminaries are presented.
\end{abstract}

\section{Hrushovski on $\omega$-CATEgorical StruCtures AND Finite LANGUAGES}

The material of this section is based on Section 7.4 of [3], pp. $353-355$ (and preliminary notes of W.Hodges). We also give some additional modifications and remarks. 
Let $N$ be a structure in the language $L$ with a unary predicate $P$. For any family of relations $\mathcal{R}$ on $P$ definable in $N$ over $\emptyset$ one may consider the structure $M=(P, \mathcal{R})$. We say that $M$ is a dense relativised reduct if the image of the homomorphism $\operatorname{Aut}(N) \rightarrow \operatorname{Aut}(M)$ (defined by restriction) is dense in $\operatorname{Aut}(M)$.

Let $L$ be the language consisting of four unary symbols $P, Q, \lambda, \rho$, a two-ary symbol $H$ and a four-ary one $S$. We will consider only $L$-structures where $P$ and $Q$ define a partition of the basic sort and $\lambda, \rho$ and $H$ are defined on $Q$. Moreover when $S(a, b, c, d)$ holds we have that $a, c \in P$ and $b, d \in Q$.

Theorem 1. If $M_{0}$ is any countable $\omega$-categorical structure then there is a countable $\omega$-categorical L-structure $N$ such that $M_{0}$ is a dense relativised reduct of $N$. In particular $M_{0}$ is interpretable in $N$ over $\emptyset$.

For every set of sentences $\Phi$ axiomatising $T h\left(M_{0}\right)$ the theory $T h(N)$ is axiomatised by a set of axioms which is computable with respect to $\Phi$ and the RyllNardzewski function of $T h\left(M_{0}\right)$.

Proof (E.Hrushovski). Let $M_{0}$ be any countable $\omega$-categorical structure in a language $L_{0}$. We remind the reader that the Ryll-Nardzewski function of an $\omega$ categorical theory $T$ assigns to any natural $n$ the number of $n$-types of $T$. So by the set $\Phi$ as in the formulation and by the Ryll-Nardzewski function of $T h\left(M_{0}\right)$ one can find an effective list of all pairwise non-equivalent formulas. Thus w.l.o.g. we may assume that $L_{0}$ is 1-sorted, relational and $M_{0}$ has quantifier elimination. In fact we can suppose that $L_{0}=\left\{R_{1}, R_{2}, \ldots, R_{n}, \ldots\right\}$ where each $R_{n}$ describes a complete type in $M_{0}$ of arity not greater than $n$. We may also assume that for $m<n$ the arity of $R_{m}$ is not greater than the arity of $R_{n}$. We admit that tuples realising $R_{n}$ may have repeated coordinates.

We now use standard material about Fraïssé limits, see [2]. Note that the class of all finite substructures of $M_{0}\left(\right.$ say $\left.\mathcal{K}_{0}\right)$ has the joint embedding and the amalgamation properties. Moreover for every $n$ the number of finite substructures of size $n$ is finite (this is the place where we use the assumption that each $R_{n}$ describes a complete type).

Let us consider structures of the language $L \cup L_{0}$ which satisfy the property that all the relations $R_{n}$ are defined on $P$. For such a structure $M$ we call a tuple $\left(a_{0}, \ldots, a_{m-1}, c_{0}, \ldots, c_{n-1}\right)$ of elements of $M$, an $n$-pair of arity $m$ if : 
(1) $m \leq n$ and $M \models \bigwedge\left\{P\left(a_{i}\right): i<m\right\} \wedge \bigwedge\left\{Q\left(c_{j}\right): j<n\right\}$;

(2) the elements $c_{i}$ are paiwise distinct and $M \models H\left(c_{i}, c_{j}\right)$ iff $(j=i+1) \bmod (n)$;

(3) $M \models \lambda\left(c_{i}\right)$ iff $i=0$ and $M \models \rho\left(c_{i}\right)$ iff $i=m-1$;

(4) $M \models S\left(a_{i}, c_{j}, a_{k}, c_{l}\right)$ iff $a_{i}=a_{j}$.

In this case we say that the $n$-pair $\bar{a} \bar{c}$ labels the tuple $\bar{a}$.

We now define a class $\mathcal{K}$ of finite $\left(L \cup L_{0}\right)$-structures as follows.

(i) In each structure of $\mathcal{K}$ all the relations $R_{n}$ are defined on $P$;

(ii) The $P$-part of any structure from $\mathcal{K}$ is isomorphic to a finite substructure of $M_{0}$;

(iii) For any $D \in \mathcal{K}$, any $n$ and any $n$-pair from $D$ labelling a tuple $\bar{a}$ we have $R_{n}(\bar{a})$.

It is obvious that $\mathcal{K}$ is closed under substructures and there is a function $f$ : $\omega \rightarrow \omega$ so that for every $n$ the number of non-isomorphic sructures of $\mathcal{K}$ of size $n$ is bounded by $f(n)$. The function $f$ is computable with respect to $\Phi$ and the Ryll-Nardzewski function.

Lemma 2. The class $\mathcal{K}$ has the amalgamation (and the joint embedding) property.

Proof. Let $D_{1}$ and $D_{2}$ be structures in $\mathcal{K}$ with intersection $C$. By induction it is enough to deal with the case where $\left|D_{1} \backslash C\right|=\left|D_{2} \backslash C\right|=1$. Let $D_{i} \backslash C=\left\{d_{i}\right\}$ and $d_{1} \neq d_{2}$. There are three cases.

Case 1. $d_{1}$ and $d_{2}$ both satisfy $P$. Using that $M_{0}$ has quantifier elimination we amalgamate the $P$-parts of $D_{1}$ and $D_{2}$ remaining the $Q$-part and $S$ the same as before. By (4) there are no new $n$-pairs in the amalgam, for any $n$.

Case 2. $d_{1}$ and $d_{2}$ both satisfy $Q$. In this case we just take the free amalgamation (without any new tuples in relations). By (4) there are no new $n$-pairs in the amalgam, for any $n$.

Case 3. $d_{1}$ satisfies $P$ and $d_{2}$ satisfies $Q$. In this case we again take the free amalgamation and by (4) we again have that there are no new $n$-pairs in the amalgam, for any $n$.

We now see that by Fraïssé's theorem, the class $\mathcal{K}$ has a universal homogeneous (and $\omega$-categorical ) structure $U$. In particular $\mathcal{K} / \cong$ coincides with $\operatorname{Age}(U)(=$ collection of all types of finite substructures of $U$ ). 
Since $M_{0}$ is the Fraïssé limit of the class of all $P$-parts of structures from $\mathcal{K}$, we see that the $P$-part of $U$ is isomorphic to $M_{0}$. Let $N$ be the reduct of $U$ to the language $L$. Note that $U$ (thus $M_{0}$ ) is definable in $N$. Indeed each $R_{n}$ is definable by the rule: $U \models R_{n}(\bar{a})$ if and only if there is an $n$-pair in $N$ which labels $\bar{a}$ (this follows from the fact that $\mathcal{K}$ contains an $n$-pair for such $\bar{a}$ ).

If two tuples $\bar{a}$ and $\bar{b}$ in $M_{0}$ realise the same type in $M_{0}$ they realise the same quantifier free type in $U$. So by quantifier elimination there is an automorphism of $U$ (and of $N$ ) which takes $\bar{a}$ to $\bar{b}$. This shows that $M_{0}$ is a dense relativised reduct of $N$.

To see the last statement of the theorem consider a set $\Phi$ axiomatising $T h\left(M_{0}\right)$. Thus the $P$-part of $U$ must satisfy $\Phi$ with respect to the relations $R_{n}$ defined in $N$ as above. The remaining axioms of $T h(N)$ (and of $T h(U)$ ) are just the axioms of the universal homogeneous structures of the corresponding class satisfying (i) (iii) as above.

Remark 3. The structure $U$ produced in the proof is axiomatised as follows.

Axiomatisation of $T h(U)$.

(a) all universal axioms forbidding finite substructures which cannot occur in $M_{0}$;

(b) all universal axioms stating property (iii) from the proof ;

(c) all $\exists$-axioms for finite substructures of $M_{0}$;

(d) all $\forall \exists$-axioms which realise the property of universal homogeneous structures that for any $\mathcal{K}$-structures $A<B$ with $A<U$ there is an $A$-embedding of $B$ into $U$.

Note that for every pair of natural numbers $n$ and $l$ the axioms of (a), (b) and (c) with at most $n$ quantifiers in the sublanguage of $L \cup L_{0}$ of arity $\leq l$ determine all $n$-element structures from $\mathcal{K}$ in this sublanguage. On the other hand by the RyllNardzewski function of $T h\left(M_{0}\right)$ we can find the arity $l_{n}$ so that all $\mathcal{K}$-embeddings between structures of size $\leq n$ are determined by their relations of arity $\leq l_{n}$. Thus the axioms of (d) with at most $n$ quantifiers can be effectively found by the corresponding axioms $(\mathrm{a}-\mathrm{c})$ and the Ryll-Nardzewski function. Moreover there is an effective procedure which for every natural numbers $n$ produces all $\forall \exists$-sentences of $T h(U)$ with at most $n$ quantifiers, when one takes as the input the axioms of (a) and (c) of $U$ with at most $n$ quantifiers. 


\section{Finite LANGUAGE AND NON-G-COMPACT THEORIES}

The following definitions and facts are partially taken from [1]. Let $\mathbf{C}$ be a monster model of the teory $T h(\mathbf{C})$. For $\delta \in\{1,2, \ldots, \omega\}$ let $E_{L}^{\delta}$ be the finest bounded $\operatorname{Aut}(\mathbf{C})$-invariant equivalence relation on $\delta$-tuples (i.e. the cardinality of the set of equivalence classes is bounded). The classes of $E_{L}^{\delta}$ are called Lascar strong types. The relation $E_{L}^{\delta}$ can be characterized as follows: $(\bar{a}, \bar{b}) \in E_{L}^{\delta}$ if there are $\delta$-tuples $\bar{a}_{0}(=\bar{a}), \bar{a}_{1}, \ldots, \bar{a}_{n}(=\bar{b})$ such that each pair $\bar{a}_{i}, \bar{a}_{i+1}, 0 \leq i<n$, extends to an infinite indiscernible sequence. In this case denote by $d(\bar{a}, \bar{b})$ the minimal $n$ such that some $\bar{a}_{0}(=\bar{a}), \bar{a}_{1}, \ldots, \bar{a}_{n}(=\bar{b})$ are as above.

Let $E_{K P}^{\delta}$ be the finest bounded type-definable equivalence relation on $\delta$-tuples. Classes of this equivalence relation are called KP-strong types. The theory $T h(\mathbf{C})$ is called $G$-compact if $E_{L}^{\delta}=E_{K P}^{\delta}$ for all $\delta$. The first example of a non-G-compact theory was found in [1. The first example of an $\omega$-categorical non-G-compact theory was found by the author in [4]. The following proposition is a straightforward application of Theorem 1

Proposition 4. There is a countably categorical structure $N$ in a finite language such that $T h(N)$ is not G-compact.

Proof. Let $L$ be defined as in the proof of Theorem[1. Corollary 1.9(2) of [8] states that G-compactness is equivalent to existence of finite bound on the diameters of Lascar strong types. Let $M_{0}$ be an $\omega$-categorical structure which is not G-compact, see 44. In 4] for every $n$ a pair $\bar{a}_{n}, \bar{b}_{n}$ of finite tuples of the same Lascar strong type is explicitely found so that $d\left(\bar{a}_{n}, \bar{b}_{n}\right)>n$.

Let $N$ be an $L$-structure, so that $M_{0}$ is a dense relativised reduct in $N$ defined by $P$. Then $T h(N)$ is not G-compact. Indeed for every $n$, the pair $\bar{a}_{n}, \bar{b}_{n}$ is of the same Lascar strong type and $d\left(\bar{a}_{n}, \bar{b}_{n}\right)>n$ with respect to the theory of $N$. To see this notice that if in $\bar{c}_{0}\left(=\bar{a}_{n}\right), \bar{c}_{1}, \ldots, \bar{c}_{m}\left(=\bar{b}_{n}\right)$ each $\bar{c}_{i}, \bar{c}_{i+1}$ extends to an indiscernible sequence in $T h\left(M_{0}\right)$, then this still holds in $T h(N)$ by density of the image of $\operatorname{Aut}(N)$ in $\operatorname{Aut}\left(M_{0}\right)$. On the other hand since $\operatorname{Aut}(N) \leq \operatorname{Aut}\left(M_{0}\right)$ on $P(M)$, we cannot find in $N$ such a sequence with $m \leq n$. 


\section{Finite language and Ehrenfeucht theories}

In this section we consider the situation where $M_{0}$ is obtained by an $\omega$-sequence of $\omega$-categorical expansions. We will see that under some natural assumptions the construction of Section 1 still works in this situation. Using this we will prove that there is a finite language such that the indexes of Ehrenfeucht theories with exactly three countable models form a $\Pi_{1}^{1}$-hard set.

Let $M_{0}$ be a countable structure of a 1-sorted, relational language $L_{0}=\left\{R_{1}, R_{2}, \ldots, R_{n}, \ldots\right\}$. Suppose $L_{0}=\bigcup_{i>0} L_{i}$, where for each $i>0, L_{i}=\left\{R_{1}, \ldots, R_{l_{i}}\right\}$ and the $L_{i}$-reduct of $M_{0}$ admits quantifier elimination (and thus $\omega$-categorical). We may assume that the arity of $R_{n}$ is not greater than $n$. Admitting $R_{n}$ with repeated coordinats, we may also assume that for all $m<n$ the arity of $R_{m}$ is not greater than the arity of $R_{n}$ and the arity of $R_{l_{i}}$ is less than the arity of $R_{l_{i}+1}$.

We now admit that $M_{0}$ is not $\omega$-categorical. On the other hand the theory of $M_{0}$ can be axiomatised as follows. For each $i$ consider the $L_{i}$-reduct of $M_{0}$ and its age $\operatorname{Age}\left(M_{0} \mid L_{i}\right)$. Then this reduct is axiomatised by the standard axioms of a universal homogeneous structure (i.e. the versions of (a),(c),(d) from Remark 3 with respect to $\left.\operatorname{Age}\left(M_{0} \mid L_{i}\right)\right)$. The collection of all systems of axioms of this kind gives an axiomatisation of $T h\left(M_{0}\right)$.

Applying the proof of Theorem 1 we associate to each $L_{i}$-reduct of $M_{0}$, a class $\mathcal{K}_{i}$ of $\left(L \cup L_{i}\right)$-structures obtained by conditions (i)-(iii) from this proof. Since the $L_{i}$-reduct of $M_{0}$ has quantifier elimination, repeating the argument of Theorem 1 we obtain an $\omega$-categorical $\left(L \cup L_{i}\right)$-structure $U_{i}$ and the corresponding $L$-reduct $N_{i}$ (since the language is finite, we do not need the assumption that each $R_{i}$ describes a type). Notice that the construction forbids $n$-pairs for $R_{n}$ of arity greater than the arity of $L_{i}$.

Lemma 5. (1) For any $i<j$ the structures $U_{i}$ and $U_{j}$ satisfy the same axioms of the form (a) - (d) of Remark 3 where the language of the P-part is restricted to $L_{i}$ and the number of variables of the $Q$-part is bounded by the arity of $L_{i}$.

(2) The corresponding structures $N_{i}$ and $N_{j}$ satisfy the same sentences which are obtained by rewriting of the axioms of statement (1) as L-sentences (using the interpretation of $U_{i}$ in $N_{i}$ ). 
Proof. Let $m$ be the arity of $L_{i}$. To see statement (1) let us prove that the classes $\mathcal{K}_{i}$ and $\mathcal{K}_{j}$ consist of the same $\left(L \cup L_{i}\right)$-structures among those with the $Q$-part of size $\leq m$. The direction $j \rightarrow i$ is clear: the $\left(L \cup L_{i}\right)$-reduct of an $\left(L \cup L_{j}\right)$-structure of this form obviously satisfies the requirements (i) - (iii) corresponding to $\mathcal{K}_{j}$ (and to $\mathcal{K}_{i}$ too). To see the direction $i \rightarrow j$ note that the assumption that the size of the $Q$-part is not geater than $m$ implies that such a structure from $\mathcal{K}_{i}$ has an expansion to an $\left(L \cup L_{j}\right)$-structure from $\mathcal{K}_{j}$.

Now the case of axioms of the form (a),(b),(c) is easy. Consider case (d). Since the $L_{i}$-reduct of $M_{0}$ admits elimination of quantifiers, for any finite $L_{j}$-substructure $A<M_{0}$ and any embedding of the $L_{i}$-reduct of $A$ into any $B \in \operatorname{Age}\left(M_{0} \mid L_{i}\right)$ there is an $L_{j}$-substructure of $M_{0}$ containing $A$ with the $L_{i}$-reduct isomorphic to $B$. This obviously implies that for any substructure $A^{\prime}<U_{j}$ without $n$-pairs for arities greater than $\operatorname{arity}\left(L_{i}\right)$, any embedding of the $\left(L \cup L_{i}\right)$-reduct of $A^{\prime}$ into any $B^{\prime} \in \mathcal{K}_{i}$ can be realised as a substructure of $U_{j}$ containing $A^{\prime}$ with the $\left(L \cup L_{i}\right)$-reduct isomorphic to $B^{\prime}$. This proves (1).

Statement (2) follows from statement (1).

We now additionally assume that $M_{0}$ is a generic structure with respect to the class $\mathcal{K}_{0}$ of all finite $L_{0}$-substructures of $M_{0}$. This means that $\mathcal{K}_{0}$ has the joint embedding and amalgamation properties $(\mathrm{JEP}$ and $\mathrm{AP}),\left(\mathcal{K}_{0} / \cong\right)=\operatorname{Age}\left(M_{0}\right)$ and $M_{0}$ is a countable union of an increasing chain of structures from $\mathcal{K}_{0}$ so that any isomorphism between finite substructures extends to an automorphism of $M_{0}$.

Let $\mathcal{K}$ be the class of all finite $\left(L_{0} \cup L\right)$-structures satisfying the conditions (i)-(iii) with respect to $\mathcal{K}_{0}$. In particular it obviously contains only countably many isomorphism types and the class $\mathcal{K}_{0}$ appears as the class of all $P$-parts of $\mathcal{K}$. Applying the proof of Theorem 1 we see that $\mathcal{K}$ is closed under substructures and has the joint embedding and amalgamation properties. By Theorem 1.5 of [6] the class $\mathcal{K}$ has a unique (up to isomorphism) generic structure (i.e. a structure which is a countable union of an increasing chain of structures from $\mathcal{K}$ and satisfies axioms (a) - (d) of Remark 3). Note that this structure can be non- $\omega$-categorical.

Lemma 6. Under the circumstances of this section let $U$ be a generic $\left(L \cup L_{0}\right)$ structure for $\mathcal{K}$ as above. 
Then the P-part of $U$ is isomorphic to $M_{0}$. The structure $M_{0}$ is a dense relativised reduct of $U$.

Proof. The firts statement is obvious. The second statement is an application of back-and-forth.

It is worth noting here that for every $m$ the amalgamation of Theorem 1 preserves the subclass of $\mathcal{K}$ consisting of structures without $n$-pairs for arities greater than $m$ (for example structures with the size of the $Q$-part less than $m+1$ ). If $m$ is the arity of the language $L_{i}$ then $\left(L_{i} \cup L\right)$-reducts of these structures form the Fraïssé class corresponding to the universal homogeneous structure $U_{i}$.

Proposition 7. (1) All axioms of $U_{i}$ of the form (a),(c),(d) of Remark 3 also hold in $U$.

(2) The theory $T h(U)$ is model complete and is axiomatised by axioms of the form (b) of Remark 3 together with the union of all axioms of the form (a),(c),(d) for all $\operatorname{Th}\left(U_{i}\right)$.

(3) For any axiom $\phi$ of $T h(U)$ of the form (a)-(d) as in (2) there is a number $i$ so that $\phi$ holds in all $U_{j}$ for $j>i$.

Proof. (1) The case of axioms of the form (a),(c) is easy. Consider case (d). Since the $L_{i}$-reduct of $M_{0}$ admits elimination of quantifiers, for any substructure $A<M_{0}$ and any embedding of the $L_{i}$-reduct of $A$ into any $B \in \operatorname{Age}\left(M_{0} \mid L_{i}\right)$ there is a substructure of $M_{0}$ containing $A$ with the $L_{i}$-reduct isomorphic to $B$. This obviously implies that for any substructure $A^{\prime}<U$ without $n$-pairs of arity greater than $\operatorname{arity}\left(L_{i}\right)$, any embedding of the $\left(L \cup L_{i}\right)$-reduct of $A^{\prime}$ into any $B^{\prime} \in \mathcal{K}_{i}$ can be realised as a substructure of $U$ containing $A^{\prime}$ with the $\left(L \cup L_{i}\right)$-reduct isomorphic to $B^{\prime}$. This proves (1).

(2) Let $U^{\prime}$ and $U^{\prime \prime}$ satisfy axioms as in the formulation of (2). Then obviously the $\left(L_{i} \cup L\right)$-reducts of $U^{\prime}$ and $U^{\prime \prime}$ satisfy the axioms of $T h\left(U_{i}\right)$ as in statement (1). In particular $P\left(U^{\prime}\right) \cong P\left(U^{\prime \prime}\right)$ in each $L_{i}$. Moreover if $U^{\prime}<U^{\prime \prime}$, then by axioms (d) one can easily verify that this embedding is $\forall$-elementary. Thus $U^{\prime}$ is an elementary substructure of $U^{\prime \prime}$ by a theorem od Robinson. It is also clear that $U$ is embeddable into any structure satisfying axioms as in (2). 
(3) By Lemma 5 we see that for every sentence $\theta \in T h(U)$ of the form (a) - (d) of (2) there is a number $i$ such that for all $j>i, \theta$ holds in $U_{j}$.

Some typical examples of Ehrenfeucht theories (i.e. with finitely many countable models) are build by the method of this section: the theory of all expansions of $(\mathbb{Q},<)$ by infinite discrete sequences $c_{1}<c_{2}<\ldots<c_{n}<\ldots$, is Ehrenfeucht and can be easily presented in an appropriate $L_{0}$ as above.

Proposition 8. Under the circumstances of this section assume that $M_{0}$ is a generic structure with respect to the class $\mathcal{K}_{0}$ of all finite substructures of $M_{0}$. Assume that $T h\left(M_{0}\right)$ is an Ehrenfeucht theory. Let $U$ be a generic $\left(L \cup L_{0}\right)$-structure for $\mathcal{K}$ as above.

Then $T h(U)$ is also Ehrenfeucht.

Proof. Let $U^{\prime}, U^{\prime \prime}$ be countable models of $T h(U)$. Assume that the $P$-parts of $U^{\prime}$ and $U^{\prime \prime}$ (say $M^{\prime}$ and $M^{\prime \prime}$ ) are isomorphic. Identifying them let us show that $U^{\prime}$ is isomorphic to $U^{\prime \prime}$. For this we fix a sequence of finite substructures $A_{1}<A_{2}<\ldots<A_{i}<\ldots$ so that $M^{\prime}=\bigcup A_{i}$. Having enumerations of the $Q$-parts of $U^{\prime}$ and $U^{\prime \prime}$ we build by back-and-forth, sequences $B_{1}^{\prime}<B_{2}^{\prime}<\ldots<B_{i}^{\prime}<\ldots$ and $B_{1}^{\prime \prime}<B_{2}^{\prime \prime}<\ldots<B_{i}^{\prime \prime}<\ldots$ with $B_{i}^{\prime}>A_{i}<B_{i}^{\prime \prime}, U^{\prime}=\bigcup B_{i}^{\prime}$ and $U^{\prime \prime}=\bigcup B_{i}^{\prime \prime}$ so that $B_{i}^{\prime}$ is isomorphic to $B_{i}^{\prime \prime}$ over $A_{i}$. By Proposition $7(2)$ using the fact that $U^{\prime}, U^{\prime \prime} \models T h(U)$ we see that such sequences exist.

We now prove that there is a finite language $L$ such that the set of Ehrenfeucht $L$-theories with exactly three models is $\Pi_{1}^{1}$-hard.

Theorem 9. There is a finite language $L$ such that for every $B \in \Pi_{1}^{1}$ there is a Turing reduction of $B$ to the set $3 M_{L} d_{L}$ of all indexes of decidable Ehrenfeucht $L$-theories with exactly three countable models.

Proof. Let $L$ be the language defined in Section 1. We use the idea of Section 4 of [7]. In particular we can reduce the theorem to the case when $B$ coincides with the index set NoPath of the property of being a computable tree $\subseteq \omega^{\omega}$ having no infinite path. The Turing reduction of this set to $3 \operatorname{Mod}_{L}$ which will be built below, is a composition of the procedure described in [10] and [7, and the construction of 
this section. The former one is as follows. Having an index $e$ of a computable tree $\operatorname{Tr}_{e} \subset \omega^{\omega}$, R.Reed defines a complete decidable theory $T_{e}$ of the language

$$
\left\langle\wedge,<_{L}, \leq_{H}, E_{\xi}^{\eta}, L_{\xi}^{\eta}, H_{\eta}, A_{\eta}, B_{\eta}, c_{\eta}\left(\eta, \xi \in T r_{e}\right)\right\rangle
$$

where $\wedge$ is the function of the greatest lower bound of a tree, $<_{L}$ is a KleeneBrouwer ordering of this tree and $\leq_{H}$ is a binary relation measuring 'heights' of nodes. Constants $c_{\eta}, \eta \in T r_{e}$, define embeddings of $T r_{e}$ into models of $T_{e}$. The remaining relations are binary.

For each natural $n$ define $\left.T_{e}\right|_{n}$ to be the restriction of $T_{e}$ to the sublanguage corresponding to the indexes from the finite subtree $\operatorname{Tr}_{e} \cap n^{<n}$. The proof of Lemma 9 from [10] shows that $\left.T_{e}\right|_{n}$ admits effective quantifier elimination. Lemma 6 of [10] asserts that every quantifier-free formula of $\left.T_{e}\right|_{n}$ is equivalent to a Boolean combination of atomic formulas of the following form:

$$
\begin{gathered}
u \wedge v=w \wedge z, u<_{L} w, u \wedge v \leq_{H} w \wedge z, E_{\xi}^{\eta}(u, w), \\
L_{\xi}^{\eta}(u, w), H_{\eta}(u \wedge v, w), A_{\eta}(u \wedge v, w),
\end{gathered}
$$

where $u, v, w, z$ is either a variable or a constant in $\left.T_{e}\right|_{n}$. By Lemma 8 of [10] the corresponding Boolean combination can be found effectively. This implies that replacing the function $\wedge$ by the first, third, sixth and seventh relations of the list above we transform the language of each $T_{e}$ into an equivalent relational language. In particular we have that each $\left.T_{e}\right|_{n}$ is $\omega$-categorical.

Note that extending the set of relations we can eliminate constants $c_{\eta}$ from our language. Admitting empty relations we may assume that all $T_{e}$ have the same language (where $\omega^{<\omega}$ is the set of indexes). Admitting repeated coordinates we may assume that this language $L_{0}=\left\{R_{1}, \ldots, R_{i}, \ldots\right\}$ satisfies the assumptions of the beginning of the section and each sublanguage $L_{n}$ of the presentation $L_{0}=\bigcup_{i>0} L_{i}$ corresponds to $\left.T_{e}\right|_{n}$.

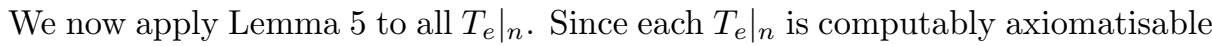
uniformly in $e$ and $n$, we obtain an effective enumeration of computable axiomatisations of $L$-expansions of all $\left.T_{e}\right|_{n}$ (with $\left.T_{e}\right|_{n}$ on the $P$-part). For each $e$ taking the axioms which hold in almost all $L$-expansions of $\left.T_{e}\right|_{n}$ we obtain by Lemma 5(1) a computable axiomatisation of a theory of $L$-expansions of $T_{e}$. 
When $T_{e}$ is an Ehrenfeucht theory with exactly three models (i.e. $e \in$ NoPath), the prime model of $T_{e}$ is generic with respect to its age. Applying Proposition 7 to $T_{e}$ and all $\left.T_{e}\right|_{n}$ we obtain a generic $\left(L_{0} \cup L\right)$-structure such that its theory is computably axiomatised as above. This theory has exactly three countable models by Proposition 8 .

When we take $L$-reducts of all $T_{e}$ and the corresponding computable axiomatisations we obtain a computable enumeration of $L$-theories which gives the reduction as in the formulation of the theorem.

Remark 10. In the proof above we used Proposition 7 in order to obtain a complete $L$-expansions of Ehrenfeuch $T_{e}$ 's. We cannot apply it in the case when $T_{e}$ does not have an appropriate generic model, for example when the corresponding $T r_{e}$ has continuum many paths. Nevertheless the author hopes that the proof can be modified so that the reduction as above also shows that the set of all $L$-theories with continuum many models is $\Sigma_{1}^{1}$-hard. In the case of infinite languages this is shown in Section 4 of [7].

\section{Coding $\omega$-CATEgorical theories}

The main theorem of this section improves the corresponding result of 7] (where the authors do not demand that the language is finite). It is worth noting that the author together with Barbara Majcher-Iwanow have found some other improvements in 5 .

Theorem 11. There is a finite language $L$ such that the property of $\omega$-categoricity distinguishes a $\Pi_{3}^{0}$-complete subset of the set of all decidable complete L-theories.

Proof. In the formulation of the theorem $L$ is the language defined in Section 1. It is shown in [7] that the property of $\omega$-categoricity is $\Pi_{3}^{0}$. The proof of $\Pi_{3}^{0}$ completeness in the case of $L$ is based on Theorem 1, Section 3 and the idea of Section 2 of [7]. The latter one will be presented in some special form, the result of a fusion with some ideas from 9 .

Let us fix the standard enumeration $p_{n}$ of prime numbers and a Gödel 1-1enumeration of the set of pairs $\langle i, j\rangle$. Let $a(x)$ be a computable increasing function 
from $\omega$ to $\omega \backslash\{0,1,2\}$ so that if natural numbers $x_{1}<x_{2}$ enumerate pairs $\left\langle i_{1}, j_{1}\right\rangle$ and $\left\langle i_{2}, j_{2}\right\rangle$ then $p_{i_{1}} a\left(x_{1}\right)<p_{i_{2}} a\left(x_{2}\right)$.

Let $L_{E}$ consist of $2 p_{n}$-ary relational symbols $E_{n}, n \in \omega$, and $T_{E}$ be the $\forall \exists$-theory of the universal homogeneous structure of the universal theory saying that each $E_{n}$ is an equivalence relation on the set of $p_{n}$-tuples which does not depend on the order of tuples and such that all $p_{n}$-tuples with at least one repeated coordinate lie in one isolated $E_{n}$-class (Remark 4.2.1 in [9]). It is worth mentioning here that the joint embedding property and the amalgamation property are easily verified by an appropriate version of free amalgamation (modulo transitivity of $E_{n}$-s). Note also that $T_{E}$ is $\omega$-categorical and decidable.

We now define an auxiliary language $L_{E S P}$. We firstly extend $L_{E}$ by countably many sorts $S_{n}, n \in \omega$. Start with a countable model $M_{E} \models T_{E}$ and take the expansion of $M_{E}$ to the language $L_{E} \cup\left\{S_{1}, \ldots, S_{n}, \ldots\right\} \cup\left\{\pi_{1}, \ldots, \pi_{n}, \ldots\right\}$, where each $S_{n}$ is interpreted by the non-diagonal elements of $M^{p_{n}} / E_{n}$ and $\pi_{n}$ by the corresponding projection. To define $L_{E S P}$ we extend $L_{E} \cup\left\{S_{1}, \ldots, S_{n}, \ldots\right\} \cup\left\{\pi_{1}, \ldots, \pi_{n}, \ldots\right\}$ by an $\omega$-sequence of relations $P_{m}, m \in \omega$, with the following properties. If $m$ is the Gödel number of the pair $\langle n, i\rangle$ then we interpret $P_{m}$ as a subset of the diagonal of $S_{n}^{a(m)}$. Let $T_{E S P}$ be the $L_{E S P}$-theory axiomatized by $T_{E}$ together with the natural axioms for all $\pi_{n}$ and $P_{m}$ as above.

Having a structure $M \models T_{E S P}$ (which is an expansion of $M_{E}$ ) we now build another expansion $M^{*}$ of $M_{E}$ (in the 1-sorted language). For each relational symbol $P_{m}$ of the sort $S_{n}^{a(m)}$ we add a new relational symbol $P_{m}^{*}$ on $M_{E}^{a(m) p_{n}}$ interpreted in the following way:

$$
M^{*} \models P_{m}^{*}\left(\bar{a}_{1}, \ldots, \bar{a}_{a(m)}\right) \Leftrightarrow M \models P_{m}\left(\pi_{n}\left(\bar{a}_{1}\right), \ldots, \pi_{n}\left(\bar{a}_{a(m)}\right)\right)
$$

It is clear that $M^{*}$ and $M$ are bi-interpretable.

By $T_{E S P}^{*}$ we denote the theory of all $M^{*}$ with $M \models T_{E S P}$. Let $L_{0}$ be the corresponding language. Then $M_{E}$ is the $L_{E}$-reduct of any countable $M^{*} \models T_{E S P}^{*}$. It is clear that $T_{E S P}^{*}$ is axiomatized by the $\forall \exists$-axioms of $T_{E}, \forall$-axioms of $E_{n^{-}}$ invariantness of all $P_{m}^{*}$ and $\forall$-axioms that every $P_{m}$ is a subset of an appropriate diagonal. Moreover for every natural $l$ we have $\leq 1$ relations of arity $l$ in $L_{0}$ and the function of arities of $P_{m}^{*}$ is increasing. Admitting empty relations (say $R_{j}$ ) we may think that for every natural number $l>0$ the language $L_{0}$ contains 
exactly one relation of arity $l$. In particular $L_{0}$ satisfies basic requirments on $L_{0}$ from Section 3. We present $L_{0}$ as the union of a sequence of finite languages $L_{1} \subset L_{2} \subset \ldots \subset L_{m} \subset \ldots$ of arities $l_{1}<l_{2}<\ldots<l_{m}<\ldots$ where $L_{m}$ consists of all relations of arity $\leq p_{n} a(m)\left(=l_{m}\right)$ with $n$ to be the first coordinate of the pair enumerated by $m$. Note that when $m$ codes a pair $\langle n, j\rangle$ the relation $E_{n}$ is also in $L_{m}$.

For every $m \in\{1, \ldots, i, \ldots, \omega\}$ and a finite set $D$ of indexes of relations $P_{i}^{*}$ of arity $\leq l_{m}$ we consider the class $\mathcal{K}_{D}$ of all finite substructures of models of $T_{E S P}^{*}$ satisfying the property that all $P_{i}^{*}$ with $i \notin D$, are empty. It is clear that for any natural number $k$ the number of structures of $\mathcal{K}_{D}$ of size $k$ is finite. We will also denote $\mathcal{K}_{\omega, D}:=\mathcal{K}_{D}$. When $m<\omega$ we define $\mathcal{K}_{m, D}$ as the class of all reducts of $\mathcal{K}_{D}$ to the sublanguage $L_{m}$.

By an appropriate version of free amalgamation we see that $\mathcal{K}_{m, D}$ has the joint embedding property and the amalgamation property. Let $M_{m, D}$ be the corresponding universal homogeneous structure and let $T_{m, D}^{*}$ be the theory of $M_{m, D}$. It follows from $T_{\omega, D}^{*}$ that $T_{E S P}^{*} \subset T_{\omega, D}^{*}$ and for every $n$ the family of all $P_{i} 1$, with $i \in D$ coding some $\langle n, j\rangle$, freely generates a Boolean algebra of infinite subsets of the sort $S_{n}$ (we may interpret such $P_{i}$ as a unary predicate on $S_{n}$ ).

By the definition of the class $\mathcal{K}_{D}$ we see that for any $t<m$ and any two finite sets $D^{\prime}$ and $D^{\prime \prime}$ satisfying

$$
D^{\prime} \cap\left\{0, \ldots, l_{t}\right\}=D^{\prime \prime} \cap\left\{0, \ldots, l_{t}\right\}
$$

the reducts of $M_{m, D^{\prime}}$ and $M_{m, D^{\prime \prime}}$ to $L_{t}$ are isomorphic.

Let us apply the construction of Theorem 1 to $M_{m, D}$. Then we obtain the $\left(L_{m} \cup L\right)$-structure $U_{m, D}$ and the corresponding $L$-reduct $N_{m, D}$, where $L$ is the language as in Theorem 1 It follows from the proof of that theorem that in the situation of the previous paragraph the structures $U_{m, D^{\prime}}$ and $U_{m, D^{\prime \prime}}$ satisfy the same axioms of the form (a) - (d) of Remark 3, where the language of the $P$-part is restricted to $L_{t}$ and the number of variables of the $Q$-part is bounded by $l_{t}$. When we rewrite these axioms as $L$-sentences (using the corresponding definition of the relations of $L_{m}$ ) we obtain that $N_{m, D^{\prime}}$ and $N_{m, D^{\prime \prime}}$ satisfy the same axioms of this kind.

\footnotetext{
${ }^{1} L_{E S P}$-predicates corresponding to $P_{i}^{*}$
} 
Let $\varphi(x, y)$ be a universal computable function, i.e. $\varphi(e, x)=\varphi_{e}(x)$. Find a computable function $\rho$ (with $\operatorname{Dom}(\rho)=\omega)$ enumerating $\operatorname{Dom}(\varphi(\varphi(y, z), x))$, i.e. the set of all triples $\langle e, n, x\rangle$ with $x \in W_{\varphi_{e}(n)}$.

For any natural $e, s$ we define a finite set $D_{e}^{s}$ of codes $m \leq l_{s}$ of all pairs $\langle n, k\rangle$ such that

$$
(\exists x)\left(\rho(k)=\langle e, n, x\rangle \wedge\left(\forall k^{\prime}<k\right)\left(\rho\left(k^{\prime}\right) \neq\langle e, n, x\rangle\right)\right) .
$$

Let $T_{e}$ and $T_{e}^{*}$ be the $L_{E S P}$-theory and the corresponding 1-sorted version (containing $\left.T_{E S P}^{*}\right)$ such that for all natural $s$ the reduct of $T_{e}^{*}$ to $L_{s}$ coincides with the corresponding reduct of $T_{s, D_{e}^{*}}^{*}$. Since for any $s<t$ we have $D_{e}^{t} \cap\left\{0, \ldots, l_{s}\right\}=D_{e}^{s}$, the definition of $T_{e}$ and $T_{e}^{*}$ is correct. It is clear that both $T_{e}$ and $T_{e}^{*}$ are axiomatisable by computable sets of axioms uniformly in $e$. Since for each $s$ the reduct of $T_{e}^{*}$ as above is $\omega$-categorical, the theories $T_{e}$ and $T_{e}^{*}$ are complete. Thus $T_{e}$ and the corresponding theory $T_{e}^{*}$ are decidable uniformly in $e$. It is worth noting that for each $m$ the $L_{m}$-reduct of $T_{e}^{*}$ admits elimination of quantifiers (it is of the form $T_{m, D}^{*}$ as above). Moreover, the class $\bigcup_{l} \mathcal{K}_{\omega, D_{e}^{l}}$ considered as a class of $L_{0}$-structures where almost all $P_{m}^{*}$ are empty, is a countable class with JEP and AP. It is clear that $T_{e}^{*}$ is the theory of the corresponding universal homogeneous structure $M_{e}^{*}$.

Applying Proposition 7 to $M_{e}^{*}$ and all $M_{l, D_{e}^{l}}$ we obtain the $\left(L_{0} \cup L\right)$-structures $U_{e}$ and their approximations $U_{l, D_{e}^{l}}$ (and $N_{l, D_{e}^{l}}$ ), which for $l \rightarrow \infty$ give a computable axiomatisation of the complete $L$-theory $T_{e}^{L}$ of the corresponding $L$-reducts $N_{e}$. By Remark 3 applied to all $U_{l, D_{e}^{l}}$ (with decidable theories), this axiomatisation (the corresponding decidability of $T_{e}^{L}$ ) can be found by an effective uniform in $e$ procedure.

We now fix a Gödel coding of the language $L$, and identify decidable complete $L$-theories with computable functions from $\left\{\operatorname{sgn}\left(\varphi_{e}(x)\right): e \in \omega\right\}$ realising the corresponding characteristic functions (by $\operatorname{sgn}(x)$ we denote the function which is equal to 1 for all non-zero numbers and $\operatorname{sgn}(0)=0)$. We want to prove that the set of all natural numbers $e$ satisfying the relation

$" \operatorname{sgn}\left(\varphi_{e}(x)\right)$ codes a decidable $\omega$-categorical theory" is $\Pi_{3}^{0}$-complete.

Fix a Turing machine $\kappa(x, y)$ which decides when for a pair $d, e$ the number $d$ codes a sentence which belongs to $T_{e}^{L}$ (in this case $\kappa(d, e)=1$ ). The following 
procedure defines a computable function $\xi(z)$ and a computably enumerable set $Z$. At step $e$ we take the Turing machine for $\operatorname{sgn}\left(\varphi_{e}(x)\right)$ and check if any replacement of some parameter $e^{\prime}$ in that program by a variable $y$ makes it the Turing machine $\kappa(x, y)$. If this happens we put $e$ into $Z$ and define $e^{\prime}:=\xi(e)$. As a result we obtain a computably enumerable set $Z$ and a computable function $\xi$ with $\operatorname{Dom}(\xi) \supset Z$ and $\operatorname{Rng}(\xi)=\omega$ such that for every $e \in Z$ the function $\operatorname{sgn}\left(\varphi_{e}(x)\right)$ is computed by the machine $\kappa(x, \xi(e))$ (for $\left.T_{\xi(e)}^{L}\right)$.

By Ryll-Nardzewski's theorem the $L_{E S P}$-theory $T_{\xi(e)}$ is $\omega$-categorical if and only if all $W_{\varphi_{\xi(e)}(n)}$ are finite (i.e. the set of 1-types (pairwise non-equivalent Boolean combinations of $P_{m}$ ) of each $S_{n}$ is finite). If we consider the corresponding $T_{f(e)}^{L}$, then this property remains true.

Since for any Turing machine computing $\varphi_{e^{\prime}}(x)$ we can effectively find a Turing machine deciding $T_{e^{\prime}}^{L}$ (i.e. in fact we can find $\operatorname{sgn}\left(\varphi_{e}(x)\right)$ with $\left.\xi(e)=e^{\prime}\right)$, we see that the $\Pi_{3}^{0}$-set $\left\{e^{\prime}: \forall n\left(W_{\varphi_{e^{\prime}}(n)}\right.\right.$ is finite $\left.)\right\}$ is reducible to $\left\{e: \operatorname{sgn}\left(\varphi_{e}(x)\right)\right.$ codes an $\omega$-categorical $L$-theory $\}$. Since the former one is $\Pi_{3}^{0}$-complete (see [7]) we have the theorem.

\section{REFERENCES}

[1] E.CASANOVAS, D.LASCAR, A.PILLAY and M.ZIEGLER, Galois groups of first order theories, J. Math. Logic, 1(2001), 305 - 319.

[2] D.EVANS, Examples of $\aleph_{0}$-categorical structures. In: R.Kaye, D.Macpherson (eds.) Automorphisms of First-Order Structures. pp. 33 - 72. Oxford University Press, 1994.

[3] W.HODGES, Model Theory, Cambridge University Press, 1993.

[4] A.IVANOV, A countably categorical theory which is not G-compact, Siberian Advances in Math., 20(2010), no.2, 75 - 82.

[5] A.IVANOV and B.MAJCHER-IWANOW, Polish group actions and computability, arXiv:0903.1070 math.LO

[6] D.W.KUEKER and C.LASKOWSKI, On generic structures, Notre Dame J. Formal Logic, 33(1992), 175 - 183.

[7] S.LEMPP and Th.SLAMAN, The complexity of the index set of $\aleph_{0}$-categorical theories and Ehrenfeucht theories, Contemporary Mathematics, vol. 425 (2007), pp. 43 - 47.

[8] L.NEWELSKI, The diameter of the Lascar strong type, Fund. Math., 176(2003), 157 - 170.

[9] R. PELAÉZ, About the Lascar group, Tesis Doctoral, (57 pgs), Departmento de Lógika, Historia y Filosofia de la Ciencia, Universidad de Barcelona, 2008. 
[10] R.REED, A decidable Ehrenfeucht theory with exactly two hyperarithmetic models, Ann. Pure Appl. Logic, 53(1991), 135 - 168.

INSTITUTE OF MATHEMATICS, UNIVERSITY OF WROCŁAW, pl.GRUNWALDZKI 2/4, 50-384 WROCEAW, POLAND

E-mail: ivanov@math.uni.wroc.pl 